duced with these standards was evaluated against the thermal expansion or phase transitions of other standards. The value of these standards and calibration methods have been proven in a number of user cases at the Diffraction User Center; and validation of these methods continues.

For further information about the Diffraction User Center see www.html.ornl.gov.

Research sponsored by the Assistant Secretary for Energy Efficiency and Renewable Energy, Office of FreedomCAR and Vehicle Technologies, as part of the High Temperature Materials Laboratory User Program, Oak Ridge National Laboratory, managed by UT-Battelle, LLC, for the U.S. Department of Energy under contract number DEAC05-00OR22725.

\section{HIGH-TEMPERATURE CAPILLARY FURNACE AT NSLS BEAMLINE x14a}

C.J. Rawn (rawncj@ornl.gov), J.Bai, M.J. Kramer, and C.R. Hubbard, Oak Ridge National Laboratory, Oak Ridge, TN 37831-6064

High temperature X-ray diffraction (HTXRD) is increasingly important in understanding materials forming and materials behavior at elevated temperatures. The Diffraction User Center at Oak Ridge National Laboratory supports several laboratory HTXRD instruments, neutron diffraction furnaces, and the X14A beam line at the National Synchrotron Light Source (NSLS). These facilities are available to users from industry and academia that propose in situ, HTXRD experiments related to improving materials to better transform or to save energy. These experiments include the measurement of thermal expansion coefficients, reaction kinetics, chemical stability, and phase transformations under a variety of atmospheres. For further information about the Diffraction User Center and user proposals see www.html.ornl.gov.

The capillary furnace recently made available at X14A is based on the Ames Laboratory design \{L. Margulies, M.J. Kramer, R.W. McCallum, S. Kycia; Rev. Sci. Instrum. 70, 3554-61 (1999)\}. Features of this furnace system are

- Temperatures from RT to $1500{ }^{\circ} \mathrm{C}$

- Rotation of the sample to increase the particle statistics

- Gas flow control system (e.g. $\mathrm{O}_{2}, \mathrm{He}, \mathrm{N}_{2} \mathrm{CO}_{2}$, Ar, or specialty gas mixtures)

- Programmable temperature controller

- Data collection with Debye-Scherrer geometry

Currently X14A is configured for high-resolution data collection via use of an analyzer crystal in the diffracted beam $\left(\mathrm{FWHM} \sim 0.015^{\circ} 2 \theta\right)$ and a scintillation detector. Medium resolution data collection is also possible. To provide much faster data collection a curved PSD detector system developed by NSLS is being tested.

Research sponsored by the Assistant Secretary for Energy Efficiency and Renewable Energy, Office of FreedomCAR and Vehicle Technologies, as part of the High Temperature Materials Laboratory User Program, Oak Ridge National Laboratory, managed by UT-Battelle, LLC, for the U.S. Department of Energy under contract number DEAC05-00OR22725.

\section{PHASE EVOLUTION OF $\mathrm{Ba}_{2} \mathrm{YCu}_{3} \mathrm{O}_{6+x}$ IN THE Ba-Y-Cu-F-O-OH SYSTEM}

Winnie Wong-Ng (winnie.wong-ng@nist.gov), I. Levin, M. D. Vaudin, L. P. Cook, J. P. Cline, Ceramics Division, NIST, Gaithersburg; and R. Feenstra, Condensed Matter Sciences Division, Oak Ridge National Laboratory, Oak Ridge, $\mathrm{TN}$

Currently, one of the most promising methods to produce long length $\mathrm{Ba}_{2} \mathrm{YCu}_{3} \mathrm{O}_{6+x}(\mathrm{Y}-213)$ coated-conductor is the "ex situ $\mathrm{BaF}_{2}$ process". This process involves the use of e-beam co-evaporated $\mathrm{BaF}_{2}-\mathrm{Y}-\mathrm{Cu}$-precursor films on rollingassisted biaxially textured metal substrates (RABiTS), followed by post-annealing in the presence of water vapor. Although the overall reaction has been determined to be

$$
\begin{aligned}
& 2 \mathrm{BaF}_{2}+1 / 2 \mathrm{Y}_{2} \mathrm{O}_{3}+3 \mathrm{CuO}+2 \mathrm{H}_{2} \mathrm{O}(\mathrm{g})- \\
& >\mathrm{Ba}_{2} \mathrm{YCu}_{3} \mathrm{O}_{6.5}+4 \mathrm{HF}(\mathrm{g}),
\end{aligned}
$$

the phase evolution and phase equilibria of the Y-213 phase in the multi-component $\mathrm{Ba}-\mathrm{Y}-\mathrm{Cu}-\mathrm{F}-\mathrm{OH}$ system are not completely understood. High-temperature x-ray experiments can provide critical information on phase evolution of Y-213 during the $\mathrm{BaF}_{2}$ post annealing process, and have been conducted on amorphous precursor $\mathrm{BaF}_{2}-\mathrm{Y}-\mathrm{Cu}$ films prepared using the e-beam evaporation technique. A theta-theta geometry $\mathrm{X}$-ray diffractometer equipped with a custom-designed vacuum and gas flow system was used for this study. With the recently installed position-sensitive-detector and the LABView software system for data collection, phase formation study of $\mathrm{Y}-213$ and $\mathrm{BaF}_{2}$ from amorphous precursor films in the presence of water vapor was conducted. This paper will discuss our recent results using the amorphous films of the $\mathrm{BaF}_{2}-\mathrm{Y}-\mathrm{Cu}-\mathrm{O}$ system and subsystems prepared at Oak Ridge National Laboratory.

\title{
Calendar of Meetings
}

\author{
Donald R. Petersen \\ Greenleaf Associates \\ 6210 Siebert Street \\ Midland, Ml 48640-2724 \\ drpetersen@voyager.net
}

\section{7-11 June 2003}

Clay Minerals Society 40th Annual Meeting. Athens, Georgia, USA. Joint meeting with the Mineralogical Society of America. [Contact: Paul A. Schroeder, University of
Georgia, Athens, GA, USA. Tel: 1 (706) 542 2384; E-mail: schroe@gly.uga.edu; Info: http://www.gly.uga.edu/ CMS2003]. 


\section{9-13 June 2003}

E-MRS 2003 European Materials Research Society Meeting. Strasbourg, France. [Contact: European Materials Research Society, 23 rue du Loess, BP 20, F-67037 Strasbourg Cedex 02, France. Tel: 33 (3) 8810 6372; Fax: 33 (3) 8810 6343; E-mail: emrs@ @ phase.c-strasbourg.fr; Info: http://www-emrs.c-strasbourg.fr].

\section{9-22 June 2003}

Twelfth Croatian-Slovenian Crystallographic Meeting. National Park Plitvice Lakes, Croatia. [Contact: Stanko Popović Tel: 385 (1) 4605 549; Info: http:// www.chem.pmf.hr/ hkz/plitvice].

\section{0-25 June 2003}

LERM 2003 International Symposium on the Role of Light Elements in Rock-forming Minerals. Nové Mêsto na Morave, Moravia, Czech Republic. Organized by the Masaryk University, Brno, Czech Republic and the University of Manitoba, Winnipeg, Canada. Technical sessions June 20-22, field trip June 23-25 to various sites in western Moravia and central Bohemia. The conference language is English. [Contact: Jan Cempirek, Department of Mineralogy, Petrology, and Geochemistry, Masaryk University, Kotlárská 2, 61137 Brno, Czech Republic. Fax: 420 (5) 4121 1214; E-mail: cempirek@sci.muni.cz; Info: http://sci.muni.cz/ $\sim$ lerm].

\section{2-26 June 2003}

Euroclay 2003. Modena, Italy. [Contact: Maria Franca Brigatti, Dipartimento di Scienza della Terra, Università di Modena e Reggio Emilia, Largo S. Eufemia 19, I-41100 Modena, Italy. Fax: 39 (059) 205 5887; E-mail: ec2003@unimo.it; Info: http://www.unimo.it/euroclay2003].

\section{2-27 June 2003}

8IKC $8^{\text {th }}$ International Kimberlite Conference. Victoria, British Columbia, Canada. [Contact: Conference Secretariat, Venue West Conference Services Ltd., 645 - The Landing, 375 Water Street, Vancouver, BC, Canada V6B 5C6. Tel: 1 (604) 681 5226; Fax: 1 (604) 681 2503; E-mail: 8IKC@ venuewest.com; Info: http://venuewest.com/8IKC].

\section{2-27 June 2003}

XAFS $12 \mathbf{1 2}^{\text {th }}$ International Conference on X-ray Absorption Fine Structure. Malmö, Sweden. Held in conjunction with the Swedish National Synchrotron Radiation Facility MAXlab, a part of Lund University. [Contact: Info: http://www.malmo-congress.com/xafs/html].

\section{2-27 June 2003}

Thin Film and Crystal Growth Mechanisms. A Gordon Research Conference. South Hadley, Massachusetts, USA. Held at Mount Holyoke College. [Contact: David G. Cahill, Department of Materials Science, University of Illinois, 1101 West Springfield Avenue, Urbana, IL 61801, USA. Tel: 1 (217) 333 6753; E-mail: d-cahill@uiuc.edu; Info: http:// www.chem.cornell.edu/ThinFilm].

\section{3-27 June 2003}

ISIC-9 Ninth International Seminar on Inclusion Compounds. Novosibirsk, Russia. [Info: http:// www.che.nsk.su/events_en/isic9].

\section{June-4 July 2003}

ICESS $99^{\text {th }}$ International Conference on Electron Spectroscopy and Structure. Uppsala, Sweden. [Contact: Ulrik Gelius, ulrik.gelius@fysik.uu.se].

\section{7-10 July 2003}

AFC 2003 Association Française de Cristallographie. Caen, France. [Contact: Secrétariat AFC 2003, Laboratoire CRISMAT, ISMRA, 6 boulevard du Maréchal Juin, 14050 Caen Cedex, France. E-mail: AFC2003@ismra.fr; Info: http://www-crismat.ismra.fr/afc2003/secretariat.htm]

\section{0-24 July 2003}

Fifteenth American Conference on Crystal Growth and Epitaxy. Keystone, Colorado, USA. Held jointly with the $\mathbf{1 1}^{\text {th }}$ Biennial Workshop on OMVPE and the $3^{\text {rd }}$ International Symposium on Laser and NLO Materials. [Contact: Info: http://www.crystalgrowth.org/conferences/ACCGE15].

\section{6-31 July 2003}

ACA'03 American Crystallographic Association Annual Meeting. Cincinnati, Ohio, USA. [Contact: Jeanette Krause Bauer, Department of Chemistry, Post Office Box 210172, Cincinnati, OH 45221-0172, USA. Tel: 1 (513) 556 9226; Fax: 1 (513) 556 9239; E-mail: jeanette.krause@uc.edu; Info: http://www.che.uc.edu/aca].

\section{July-2 August 2003}

Microscopy and Microanalysis 2003. San Antonio, Texas, USA. Sponsored by the Microscopy Society of America and the Microbeam Analysis Society. [Contact: MSA Business Office, Bostrom Corporation, 230 East Ohio, Suite 400, Chicago, IL 60611, USA. Tel: 1 (312) 644 1527; Fax: 1 (312) 644 8557; E-mail: businessoffice@msa.microscopy.com; Info: http://www.msa.microscopy.com]

\section{4-8 August 2003}

Denver X-ray Conference. Denver, Colorado, USA. Plenary session: "X-ray Studies of Art and Archeological Objects." [Contact: Terry Maguire, Conference Administrator, International Centre for Diffraction Data, 12 Campus Boulevard, Newtown Square, PA 19073-3273, USA. E-mail: maguire@icdd.com; Info: http://www.dxcicdd.com].

\section{0-13 August 2003}

AsCA'03/CRYSTAL 23 Joint meeting of the Asian Crystallographic Association and the Society of Crystallographers in Australia and New Zealand. Broome, Western Australia, Australia. [Contact: Syd Hall, Crystallography Centre, University of Western Australia, Nedlands 6009, WA, Australia. Tel: 61 (8) 9380 2725; Fax: 61 (8) 9380 1118; E-mail: srh@crystal.uwa.edu.au; Info: http:// www.crystal.uwa.edu.au/CrystalsDownUnder]. 
24-29 August 2003

ECM-21 $21^{\text {st }}$ European Crystallographic Meeting. Durban, South Africa. [Contact: Prof. Andreas Roodt, Free State University. E-mail: RoodtA@sci.uovs.ac.za; Info: http:// www.ecm21-africa.co.za].

\section{5-29 August 2003}

SRI 2003 Eighth International Conference on Synchrotron Radiation Instrumentation. San Francisco, California, USA. [Contact: Jeffrey Troutman, Advanced Light Source, MS6R2100, 1 Cyclotron Road, Berkeley, CA 94720-8235, USA. Tel: 1 (510) 495 2001; Fax: 1 (510) 486 4773; E-mail: sri03@1bl.gov; Info: http://www.sri2003.lbl.gov].

\section{August-4 September 2003}

XIX Conference on Applied Crystallography. Krakow, Poland. For those with an interest in structure studies applied to materials used in industrial practice. The conference chairman will be Prof. Henryk Morawiec of the University of Silesia. This meeting is followed directly by the Summer School on Polycrystalline Structure Determination by Direct Methods (4-7 September), under Prof. Carmelo Giacovazzo of CNR, Bari, Italy. [Contact: Dr. Danuta Stró[FOFC], University of Silesia, Institute of Physics and Chemistry of Metals, ul. Bankowa 12, 40-007 Katowice, Poland. Tel/Fax: 48 (32) 5969 29; E-mail: dana@us.edu.pl; Info: www.us.edu.pl/uniwersytet/konferencje/2003/cac/tekst/ index.shtml].

\section{3-6 September 2003}

ECNS 2003 Third European Conference on Neutron Scattering. Montpellier, France. [Contact: Nellie Malartic, ECNS 2003 Conference Office, Université Montpellier II, Case 069, F-34095 Montpellier Cedex 5, France. E-mail: info@ecns2003.org; Info: http://ecns2003.org].

7-12 September 2003

$\mathbf{1 3}^{\text {th }}$ Goldschmidt Conference. Kurashiki, Japan. The premier annual meeting for geochemistry and geochemical processes. [Contact: Goldschmidt 2003 Secretariat, International Communications Specialists, Inc., Sabo Kaikan Bekkan 2-7-4 Hirakawa-cho Chiyoda-ku, Tokyo 102-8646, Japan; Tel: 81 (3) 3263 6474; Fax: 81 (3) 3263 7537; E-mail: gold2003@ics-inc.co.jp; Info: http://www.ics-inc.co.jp/ gold2003].

\section{8-13 September 2003}

Aperiodic 2003. Belo Horizonte, Minas Gerais, Brazil. [Contact: Secretaria do Aperiodic 2003, Departamento de Fisica, Universidade Federal de Minas Gerais, CP 702, Belo Horizonte 30123-970, Minas Gerais, Brazil. Tel: 55 (31) 3499 5672; Fax: 55 (31) 3499 5600; E-mail: ap2003@fisica.ufmg.br; Info: http://www.fisica.ufmg.br/ $\sim$ ap2003].

\section{4-19 September 2003}

SSPD'03 Structure Solution from Powder Diffraction Data. Stara Lesna, Slovakia. [Contact: Dr. Lubomir Smrcok, Institute of Inorganic Chemistry, Slovak Academy of Sciences, Dubravska cesta, SK-842 36 Bratislava, Slovak Republic. Fax: 421 (2) 5941 0444; E-mail:
Lubomir.Smrcok@savba.sk; Info: http://www.sspd03.sav.sk].

\section{5-19 September 2003}

XV International Conference on X-ray Diffraction and Crystal Chemistry of Minerals. St. Petersburg, Russia. [Contact: Dr. M. G. Krzhizhanovskaya, Department of Crystallography, St. Petersburg State University, University Emb. 7/9, St. Petersburg 199034, Russia. E-mail: xrd2003@ crystal.pu.ru; Info: http://www.lcm3b.u-nancy.fr/ cims/forthcoming.htm].

\section{2-25 September 2003}

81. Jahrestagung der Deutschen Mineralogischen Gesellschaft. Bochum, Germany.

\section{2-26 September 2003}

$17^{\text {th }}$ International Congress on X-ray Optics and Microanalysis. Chamonix Mont-Blanc, France. [Contact: ICXOM XVII, ESRF, Post Office Box 220, F-38043 Grenoble, France. Tel: 33 (476) 882 438; Fax: 33 (476) 882 542; E-mail: icxom@esrf.fr; Info: http://www.esrf.fr/ conferences/icxom.html].

\section{1-5 December 2003}

Fall Meeting of the Materials Research Society. Boston, Massachusetts, USA. [Contact: Materials Research Society, 506 Keystone Drive, Warrendale, PA 15086-7573, USA. Tel: 1 (724) 779 3003; Fax: 1 (724) 779 8313; E-mail: info@mrs.org; Info: http://www.mrs.org].

\section{4-18 March 2004}

$\mathbf{1 3 3}^{\text {rd }}$ Annual Meeting of the Minerals, Metals \& Materials Society. Charlotte, North Carolina, USA. [Contact: TMS, 184 Thorn Hill Drive, Warrendale, PA 15086, USA. Tel: 1 (724) 776 9000; Fax: 1 (724) 776 3770; E-mail: ckobert@tms.org; Info: http://www.tms.org/Meetings/ Annual-04/AnnMtg04Home.html].

\section{7-22 July 2004}

ACA'04 American Crystallographic Association Annual Meeting. Chicago, Illinois, USA. [Contact: Bernie Santarsiero, bds@uic.edu; Karl Volz, kvolz@uic.edu; Info: http:// www.hwi.buffalo.edu].

\section{2-5 September 2004}

EPDIC 9 Ninth European Powder Diffraction Conference. Prague, Czech Republic. Held at Charles University. [Contact: E-mail: kuzel@karlov.xray.cz/epdic; Info: http:// www.xray.cz.epdic].

\section{3-31 August 2005}

$20^{\text {th }}$ IUCr General Assembly and International Congress of Crystallography. Florence, Italy. [Contact: Carlo Mealli. E-mail: mealli@fi.cnr.it; Info: http://www.iucr2005.it].

28 November-2 December 2005

International Conference on Neutron Scattering. Sydney, Australia. 